\title{
Frequency dependence of dielectric properties of ex situ MgB2 bulks
}

\begin{abstract}
In this study, frequency dependent electrical properties of ex situ polycrystalline $\mathrm{MgB} 2$

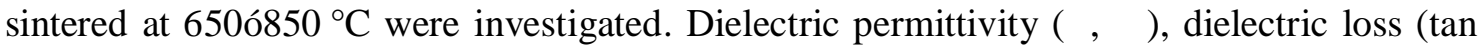
$\breve{\mathrm{u}}$ ), alternating current (AC) conductivity (Ůac) as a function of frequency (100 Hzï $10 \mathrm{MHz}$ ) were measured at room temperature. The X-ray diffraction (XRD) and grain morphology were analysed and correlated to the findings in dielectric properties. Due to weakly coupled grains and presence of high fraction of oxides, positive real dielectric permittivity was measured for the ex situ samples as compared with the negative real dielectric permittivity shown by the in situ MgB2. Nevertheless, the samples sintered at higher temperature showed improved grain connectivity as reflected by the higher AC conductivity and dielectric loss. The semicircle observed in the complex impedance plots together with the combined spectroscopy plots indicates that the electrical behavior of the ex situ samples is mainly due to the bulk and grain boundary responses as opposed to the sole bulk response of the in situ $\mathrm{MgB}$ 2. The modelled equivalent circuit also suggests the presence of insulating grain boundary barrier (due to the oxide phases) next to the conducting bulk in the ex situ samples.
\end{abstract}

Keyword: MgB2; Dielectric properties; Frequency dependence 